\title{
X-RAY ABSORPTION NEAR EDGE SPECTRA FOR CdTe - THEORETICAL STUDY
}

\author{
J. Oleszkiewicz, R. MaRKowski ANd A. Kisiel \\ Institute of Physics, Jagiellonian University, Reymonta 4, 30-059 Kraków, Poland
}

\begin{abstract}
The theoretical band structure of the CdTe was calculated using conventional, $a b$ initio, self-consistent, relativistic LMTO method. The projected density of states and the dielectric function were produced within the local density approximation. Spin-orbit coupling was included in the perturbation approach. X-ray absorption spectra for the $\mathrm{Cd} L_{1,3}$ - and Te $L_{1,3}$-edges were calculated and the results compared with experimental data. The teoretical results were obtained from the calculations with inclusion of $k$-dependent transition matrix elements, spin-orbit coupling, extended set of basis functions and finite lifetime of initial and final states. They were compared with other calculations which use common constant matrix element approximation. The work presents final conclusion concerning validity of using constant matrix element approximation within one-electron scheme.
\end{abstract}

PACS numbers: 78.70.Dm, 78.20.Bh, 78.20.Dj

\section{XANES measurements}

The X-ray absorption measurements have been carried out with the use of synchrotron radiation at the ADONE WIGGLER facility in Frascati [1] using a $\mathrm{Si}(111)$ channel-cut crystal monochromator. The crystalline samples were kindly provided by the Institute of Physics of Polish Academy of Sciences in Warsaw. The samples were powdered and deposited on polyacetate films as required for XANES measurements. The resulting instrumental Gaussian broadening has been estimated to about $0.7 \mathrm{eV}$ for Te and $\mathrm{Cd} L$-edges. The contribution of each edge to the absorption coefficient has been extracted by extrapolating the pre-edge spectrum to higher energies by a Victoreen fit and by substracting the fitted curve from the remaining experimental spectrum [2]. The whole set of the experimental data for CdTe has been presented in another paper [3]. The standard experimental data reduction method has been used [3]. 


\section{Electronic structure calculations}

The bands and density of states have been obtained using the self-consistent, semirelativistic LMTO method $[4,5]$. The exchange-correlation local spin density approximation (LSDA) potential was used in the form proposed by Vosko, Wilk and Nusair [6]. The openness of the zinc-blende structure was dealt with in the standard way by placing additional "empty spheres" into the unit cell. The calculations were carried out using the experimental lattice constant. The results of such band structure calculation formed a basis for X-ray absorption spectra calculation.

\section{X-ray absorption spectra calculations}

According to the simple atomic-based selections rules the $\mathrm{L}_{1}$-edge is due to the electronic dipole transitions from the $2 s$ core level to $p$-like conduction states and the $L_{2,3}$-edges are due to transitions from $2 p$ core levels to $s$-like and $d$-like conduction states. The probabilities of the electronic quadrupole transitions $(\Delta l=0, \pm 2)$ for the Te and $\mathrm{Cd} L_{1,3}$-edges are negligible [7]. The dipol transition matrix elements have been calculated by the method presented by Koenig, Khan [8] and by Alouani et al. [9] and implemented by Markowski and Podgórny [10]. In the one-electron approximation the X-ray absorption coefficient from a core level (c) to final unoccupied conduction states (f) is given by the expression:

$$
\mu_{0}(\hbar \omega)=\text { const } \sum_{f} \int_{B Z} \mathrm{~d}^{3} k\left|M_{f c}^{k}\right|^{2} \delta\left(E_{f}^{k}-E_{c}-\hbar \omega\right),
$$

where

$E_{f}^{k}$ is an empty state energy at a given $k$ vector of the final states band,

$E_{c}$ is the core state energy with the negligible energy dispersion through the Brillouin zone (BZ) and well-defined angular momentum $l$,

$M_{f c}^{k}$ stands for the $\boldsymbol{k}$-dependent dipolar matrix element.

It is known that it is possible to write several equivalent expressions for the matrix element. This equivalence is just only for the exact wave functions, which are solutions of the Schrödinger equation in the whole Hilbert space. In the band structure method, the approximate wave functions are solutions in the subspace spanned by the finite number of basis functions. We have chosen the dipolar matrix element in the form:

$$
M_{f c}^{k}=\left\langle f k\left|\frac{\hbar \nabla}{\mathrm{i}}\right| c\right\rangle
$$

where

c means the core level with the well-defined $n, l$ quantum numbers,

$|f k\rangle$ is the wave function obtained from scalar-relativistic self-consistent LMTO scheme.

The dipole approximation picks out the final states of symmetry $l \pm 1$ which are coupled to the initial state of symetry $l$. The final conduction states have the 
finite energy-dependent lifetime $\Gamma_{x}$, so the Müller correction has been taken into account using the formula [11]:

$$
\mu^{\prime}(E)=\frac{1}{2 \pi} \int_{-\infty}^{+\infty} \frac{\mu_{0}\left(E^{\prime}\right) \Gamma_{x}\left(E^{\prime}\right)}{\left(E^{\prime}-E\right)^{2}+\Gamma_{x}\left(E^{\prime}\right)^{2} / 4} \mathrm{~d} E^{\prime} .
$$

The absorption coefficient $\mu^{\prime}(E)$ has been finally convoluted with Gaussian and Lorentzian functions. The Gaussian function reflects the instrumental resolution and the Lorentzian function reflects the core-level lifetime:

$$
\begin{aligned}
& \mu^{\prime \prime}(E)=\int_{-\infty}^{+\infty} \mu^{\prime}\left(E^{\prime}\right) L\left(\Gamma_{\mathrm{L}}, E^{\prime}-E\right) \mathrm{d} E^{\prime}, \\
& L(\Gamma, E)=\frac{1}{2 \pi} \frac{\Gamma}{E^{2}+\Gamma^{2} / 4}
\end{aligned}
$$

and finally

$$
\bar{\mu}(E)=\frac{1}{\sqrt{2 \pi} \Gamma_{\mathrm{G}}} \int_{-\infty}^{+\infty} \exp \left[-\frac{\left(E-E^{\prime}\right)^{2}}{2 \Gamma_{\mathrm{G}}^{2}}\right] \mu^{\prime \prime}\left(E^{\prime}\right) \mathrm{d} E^{\prime} .
$$

The value of the Lorentzian width $\Gamma_{\mathrm{L}}$, which reflects the core-level lifetime, was optimized to obtain the best agreement of the theoretical spectra with the experimental ones in the surrounding of the absorption edge, see Table (about $8 \mathrm{eV}$ around the first inflection point of the edge).

\section{TABLE}

The result of the fitting $\Gamma_{\mathrm{L}}$ core-level width.

\begin{tabular}{c|c|c}
\hline \hline & $\mathrm{Cd}$ & $\mathrm{Te}$ \\
\hline$L_{1}$ & $2.0 \pm 0.1$ & $2.1 \pm 0.1$ \\
\hline$L_{3}$ & $2.4 \pm 0.1$ & $2.4 \pm 0.1$
\end{tabular}

\section{Discussion}

Figures 1-3 show the results for the $\mathrm{Te} L_{3}, \mathrm{Cd} L_{1}$ and $\mathrm{Cd} L_{3} \mathrm{X}$-ray absorption edges. Case A represents the constant transition matrix approximation, and case $B$ represents the calculation with $k$-dependent dipole transition matrix. We find that for the $\mathrm{Cd} L_{1}$ and Te $L_{3}$ the energy dependence of the transition matrix element is so weak that the constant transition matrix approximation seems to be good enough. The $L_{2,3}$-edges arise from a superposition of the transitions to $s$-like and $d$-like conduction states. The constant matrix element approximation causes an additional problem of the value of the ratio between the matrix elements of $s$ and $d$-like states. We must assume arbitrarily a certain value for the $k$-independent 


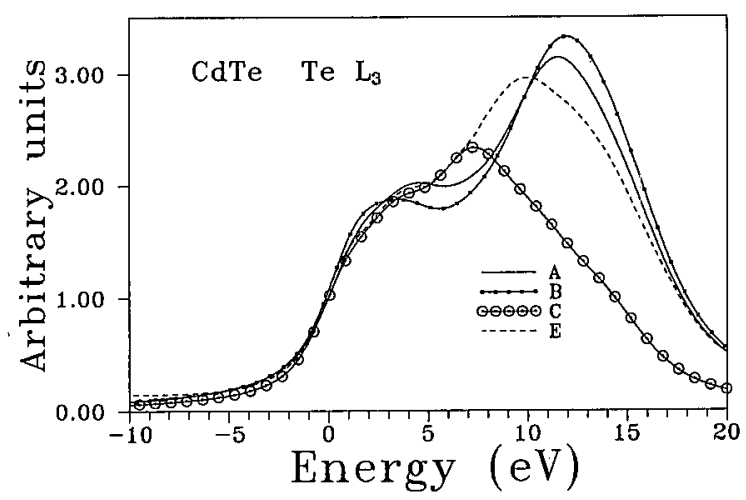

Fig. 1. The calculated Te $L_{3} \mathrm{X}$-ray edge spectra for CdTe. The curve A - constant transition matrix approximation, B - calculations with full transition matrix element, $\mathrm{C}$ - calculation with full transition matrix element and extended basis function set and E-experimental spectrum.

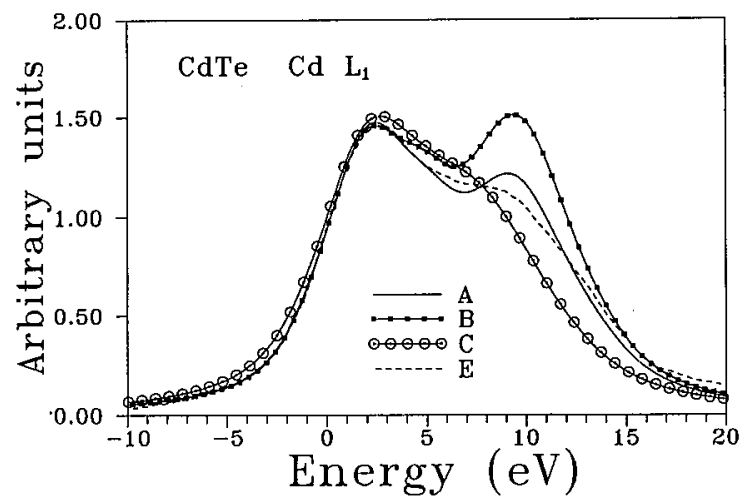

Fig. 2. The calculated Cd $L_{1}$ X-ray edge spectra for CdTe. The curve A - constant transition matrix approximation, $\mathrm{B}$ - calculations with full transition matrix element, $\mathrm{C}$ - calculation with full transition matrix element and extended basis function set and E-experimental spectrum.

matrix elements. We assume equivalent weight for both transition in the case $A$ of the calculations. The curves A and B do not fit totally with experimental data for the Cd $L_{3}$-edge. The inclusion of transition matrix elements changes drastically the calculated shape, but still there exist big disagreements between theory and experiment. The main reason is the fact that the LMTO method uses a finite basis function set. In the presented calculations, the basis function set includes the $4 d$ $\mathrm{Cd}$ functions but does not include the $5 d \mathrm{Cd}$ functions. The $4 d \mathrm{Cd}$ functions describe the $4 d \mathrm{Cd}$ states lying about $10 \mathrm{eV}$ below the valence band maximum. These states have a significant influence on the nature of the valence bands and 


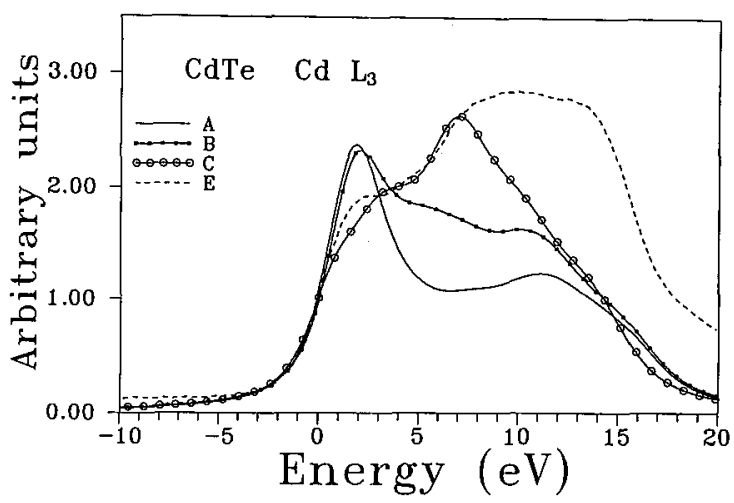

Fig. 3. The calculated $\mathrm{Cd} L_{3} \mathrm{X}$-ray edge spectra for CdTe. The curve A - constant transition matrix approximation, $B$ - calculations with full transition matrix element, $\mathrm{C}$ - calculation with full transition matrix element and extended basis function set and E-experimental spectrum.

the bottom of the conduction states. The center of the $5 d \mathrm{Cd}$ states is located about $10 \mathrm{eV}$ above the conduction band minimum. The $4 d$ and $5 d$ Cd states have the same crystallographic symmetry, so the presence of these states together in the basis function set will cause mathematics-computation complications. This is the reason that the $5 d$ Cd states have been removed from the basis function set. Therefore we made another calculation with an extended basis function set including the $5 d \mathrm{Cd}$ states instead of the $4 d \mathrm{Cd}$ states. This is the case $\mathrm{C}$ in Figs. 1-3. We can see that now the agreement between theoretical and experimental spectra for $\mathrm{Cd} L_{1,3}$ is much better. But there exists now a bigger disagreement for the Te $L_{1,3^{-}}$-edges. This is due to the hybridization effects between $\mathrm{Cd}$ and $\mathrm{Te}$ states and due to the fact that this new basis function set overestimates the weight of the states lying very high above the Fermi level. In general we find that the one-electron approximation is quite good for a theoretical description of the X-ray edges.

\section{Acknowledgments}

This work was supported in part by the Polish Central Research Program CPBP 01.12 .

\section{References}

[1] E. Burattini, E. Bernieri, A. Balerna, C. Menuccini, R. Rinzivillo, G. Dalba, P. Fornasini, Nucl. Instrum. Methods Phys. Res. A 246, 125 (1986).

[2] B.T. Teo, EXAFS: Basic Principles and Data Analysis, Springer, Berlin 1986.

[3] A. Kisiel, G. Dalba, P. Fornasini, M. Podgórny, J. Oleszkiewicz, E. Burattini, Phys. Rev. B 39, 7895 (1989). 
[4] O.K. Anderson, Phys. Rev. B 12, 3060 (1975).

[5] H.L. Skriver, The LMTO Method, Vol. 41, in series of Solid State Sciences, Springer, Berlin 1984.

[6] S.H. Vosko, L. Wilk, M. Nusair, Can. J. Phys. 58, 1200 (1980).

[7] J.E. Müller, J.W. Wilkins, Phys. Rev. B 29, 4331 (1984).

[8] C. Koenig, M.A. Khan, Phys. Rev. B 27, 6129 (1983).

[9] M. Alouani, J.M. Khoch, M.A. Khan, J. Phys. F 16, 473 (1986).

[10] R. Markowski, M. Podgórny, J. Phys. Condens. Matter 4, 2505 (1990).

[11] J.E. Müller, O. Jepsen, J.W. Wilkins, Solid State Commun. 42, 365 (1982). 